\title{
A sustainability-driven approach for participatory modelling
}

\author{
Michel LAMPLE $^{\mathrm{a}}$, Denis BAILLY ${ }^{\mathrm{a}}$ and Johanna BALLE-BEGANTON ${ }^{\mathrm{a}}$ \\ ${ }^{a}$ Université de Brest, UEB, UMR Amure, 12 rue de Kergoat, 29238 Brest Cedex 3, France Email: \\ lample@univ-brest.fr
}

\begin{abstract}
We analyse how to improve participatory modelling aimed at addressing complex socioecological problems when it is driven by one of the actors involved. Whether they are stakeholders, modellers, decision-makers or even researchers, such "actor-driven" approaches lead to significant drifts in the process of engagement and have raised many doubts about the contribution of modelling. The sustainability-driven approach presented here is a method based on a qualitative Agenda, with emphasis on the exploration of the problem and a slow growing quality in model development to reduce the biases imposed by solution finding finality. A Design-Formulation-Output-Appraisal iterative protocol, separate from the Agenda for model development, is proposed to sustain the interactions between modellers and other participants, as well as communication among them. This is to answer the need to maintain oscillations between modellers and stakeholders and to offer a quality context able to avoid the drift of the process. The sustainability-driven method also promotes the need for an Architect that is not only a project management or facilitation role, but has to be a driving force for collective engagement in participatory modelling.
\end{abstract}

Keywords: $\quad$ Sustainability, Co-construction, Participatory modelling, System modelling. 


\section{DRAWBACKS OF ACTOR-DRIVEN APPROACHES IN PARTICIPATORY MODELLING}

In the present context of complex socio-ecological issues, participatory modelling techniques are highly recommended. Voïnov et al. (2010) emphasised that "hardly any environmental assessment or modelling effort today can be presented without some kind of reference to stakeholders". The different modelling approaches (policy-issue vs. scientific-question oriented, modellers vs. stakeholders/managers driven) can be seen as variations of attempts of the scientific community to "sell" its expertise in the arena of public policy. Whichever approach is retained, the idea of participatory modelling is not delivering its promises. The literature doesn't show a clear success story of the many methodologies and platforms that has been tried. In this paper, we argue that a sustainability-driven method is needed to reconcile the objectives of inclusiveness and efficiency.

\subsection{Actor-driven approaches}

According to Voïnov et al (2010), "it is generally agreed that better decisions are implemented with less conflict and more success when they are driven by stakeholders, that is by those who will bearing their consequences". However participation of stakeholders may not be enough to ensure the effectiveness of coconstructed studies in terms of support to public policy. For example, Gregory (1993) points out the problem of insufficiency of the classical Soft System Methodology (SSM) developed by Checkland (1981). Its main feature is the construction of a common conceptual model through an iterative debate driven by members of the client organization (stakeholders). As showed by Gregory (1993), the conceptual model only includes "necessary conditions" as discussed by stakeholders. Gregory explains that "a physical system that is based on a model that contains only necessary conditions can never be guaranteed to work. It may work because the necessary conditions may in fact also be sufficient but it is also possible that they might not be". Gregory continues by noting that "in all traditional SSM models, the arrows in the model are meant to be represented by necessary conditions". Indeed, we noted in the experiment SPICOSA (BalleBeganton et al. 2010) that the stakeholders took care to point out only the necessary conditions for their activities (while learning with interest their downstream impacts). Applying the analysis of Gregory, we realize that participatory approaches are biased in many instances by necessary conditions of other participants:

- A "modelling-driven" or "modeller-led" protocol assumes that numerical models are the way to solve environmental problems. We know that it is not a sufficient condition. But we can even question when a numerical model is or is not a "necessary" condition to deal with complex socioecological issues.

- A "policy-driven" or "manager-led" oriented approach is expected to lead to decisions. So this imperative will often offset other necessary conditions such as understanding the complexity of the problem. For example, regarding the problem of eutrophication and green algae (nitrogen excess), many managers and technology providers' advocate for biogas production. This is typically a "decision imperative" that leads such discourse "something must be done". However, since biogas is mainly composed of carbon and oxygen, the problem of nitrogen excess will only be displaced.

- A "research-driven" or "researcher-led" work is expected to publish advances in science. But is publication a necessary condition to enlighten policy debate by scientific knowledge? This is a necessary condition only for the scientist.

To avoid these biases we must go back to the basic thread of environmental management which is sustainability. What could be the key characteristics of a "sustainability-driven approach"? A first step to avoid the common drawbacks of actors driven approaches is to adopt a project management strategy including the importance of leadership and engagement methodology. In terms of leadership, a new role appears: the one of an Architect which role is only to set the sustainability stakes in order to build a common view upon the policy makers concerns, the stakeholders' claims, the scientist knowledge and the modelling potential. The methodology must be set to avoid the drifts commonly found in participatory modelling.

\subsection{The drifts of co-construction}

Janssen et al. (2009) remarks that "a gap between the model and its users is unavoidable; model applications are dominated by the fairly technical conceptions which modellers have of the system, and fail to address high level construals brought up by the stakeholders". Bridging the gap between scientists and stakeholders has become a major concern of co-construction and is well described by McIntosh et al (2008). However, rather than the gap itself, let us examine the drift of the process. Along the process, the model tries to meet the stakeholders' expectations but meanwhile, the stakeholders' perception of the issue might evolve towards a very different target (figure 1). This is a "mutual pursuit" involving modellers and 
stakeholders. The rationale underlying the divergence of such pursuit (drift) is given by two factors: the fuzzy perception of the target and the gap between 'theory-in-use' \& 'espoused theory' in building the models.

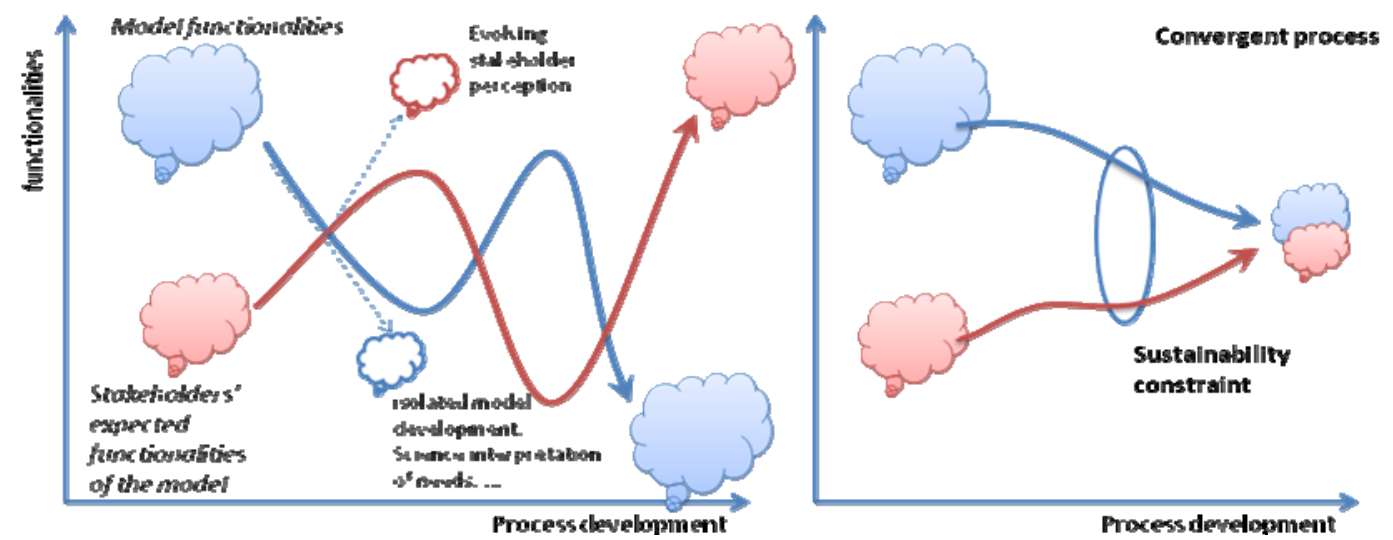

Figure 1. Divergent process (left) due to a mutual uncontrolled pursuit and Convergent process (right) decreasing the gap between models and expected functionalities of the models by stakeholders.

Fuzzy perception of the target: from the problem to the need

In order to examine the problem of fuzzy targets, it is interesting to study experiences gained from some commercial practices. Mike Bosworth (1993) observed his customers face problems they believe have no solution. Usually, they unconsciously bury them deeply. Businessmen have to analyse and understand the problems of their customer in order to bring to light the possibility of a solution (of course, a solution he can provide). The lesson learned from this business practice is that finding a solution should not be considered as the first stage of environmental management. The construction of a solution is only an engineering process. Before this stage, we must very carefully consider that there is first a "problem stage" and a long way from the problem to the recognition of a need for solution.

A socio-ecological problem can simply consist of an aggregate of claims from different actors, the necessary conditions of Gregory, like the problem of building a house supposed to meet the requirements of parents, children, neighbours, banks, local rules... The accumulation of requirements, all necessary conditions, may lead to a seemingly unsolvable problem. At this point, the actors agree that the help of an architect is welcome. The first role of the architect is to lead the actors from this "problem stage" to an acceptable definition of the "common need". This in the first instance is a 2D or 3D drawing which will be instrumental on the way to the "solution", the future house. Ignoring the importance of the step "problem stage" to "common need" and moving straight to the "solution" is a major weakness of many participatory processes.

From the problem to the need, there is a real investigation to be done, not as solution sellers usually do, i.e. starting from the problem and not from a pre-defined solution. In that initial stage, the quality of the participatory process (inclusiveness, with all time needed) is crucial in search of such "well posed" problem. Setting the problem in terms of sustainability stakes and associated social claims, may help to clarify the target beyond the fuzziness of the initial problem.

The gap between "theory-in-use" and "espoused theory"

The "espoused theory" is a concept developed by Argyris (1999). He explains that the maps the modellers use to build their model are not the theories they explicitly espouse (particularly during stakeholders meeting). So the result of their modelling process is the fact of a "theory-in-use" often different from the so called "espoused theory". Besides, the stakeholders draw themselves their own picture from the modellers' outcomes and this, with their own "theory-in-use" which can be different from their ordinary "espoused views of the world". This partly relates to the role game induced by the collaborative scene. The two gaps that can be interpreted as "cognitive dissonances" are maintained along the collaborative process by the "necessary conditions" of each actor. Each actor may promote its own view of the system in regard of his expertise: "The issue needs what I can provide". These "necessary conditions" weave a web of beliefs that it is necessary to unwind. But, beyond good intentions, it is difficult to change beliefs. Burgelman (1994) says that there is a real challenge to convince partners that 'Self-evident truths' are no longer true'.

These two reasons are the major amplifying factors in the modellers-stakeholders pursuit problem. The drift is the divergence of the co-construction process and it lies in the increase of the gap between the output of 
the process, the model, and stakeholders" expectations. This can explain the "stakeholder fatigue" observed by many authors. L'Etang (2006) claims that "Stakeholder fatigue occurs when organization solicits too much contact with key publics". But the European project SPICOSA (Science and Policy Integration for COastal System Assessment www.spicosa.eu) has shown that this fatigue can also be due to a drift in the collaborative process. Either because of target remaining fuzzy or because of an impossible unwind of the "theories-in-use" (e.g. managers' belief about the solutions they promote, stakeholders' belief about the needs for their activities) and the gap with espoused theories or views of the world. The finality of a sustainability-driven approach is to avoid such drifts in order to maintain the engagement and avoid participants' defection.

\section{THE SUSTAINABILITY-DRIVEN APPROACH}

\subsection{From identified needs to realization}

From the start, attempts to offer efficient methodologies for models development have been enthusiastically received. The need for that was enormous given the inability of the software industry to provide efficient support. After the first PERT charts developed for Navy projects in the 1950s, the Waterfall approach of Benington (in a symposium of 1956, next republished in 1983) has been widely used by the modelling community. The Waterfall approach is linear. The project goes from phase to phase in a sequential manner. It defines the transition from analysis to code into more or less six stages (figure 2). Rapidly the Waterfall method showed its weaknesses. It falls apart when the problem changes due to requirement modifications. New approaches emerged as the "V" method proposing iterative or incremental processes. In the Spiral methodology (Boehm (1986) the model is "incremented" at each iteration with additional planning, risk assessment, prototyping... It is an iterative process but inherits the main drawbacks of the Waterfall model. Even the "brute force" method "code and fix" belongs to this iterative category as well as RUP, AGILE...

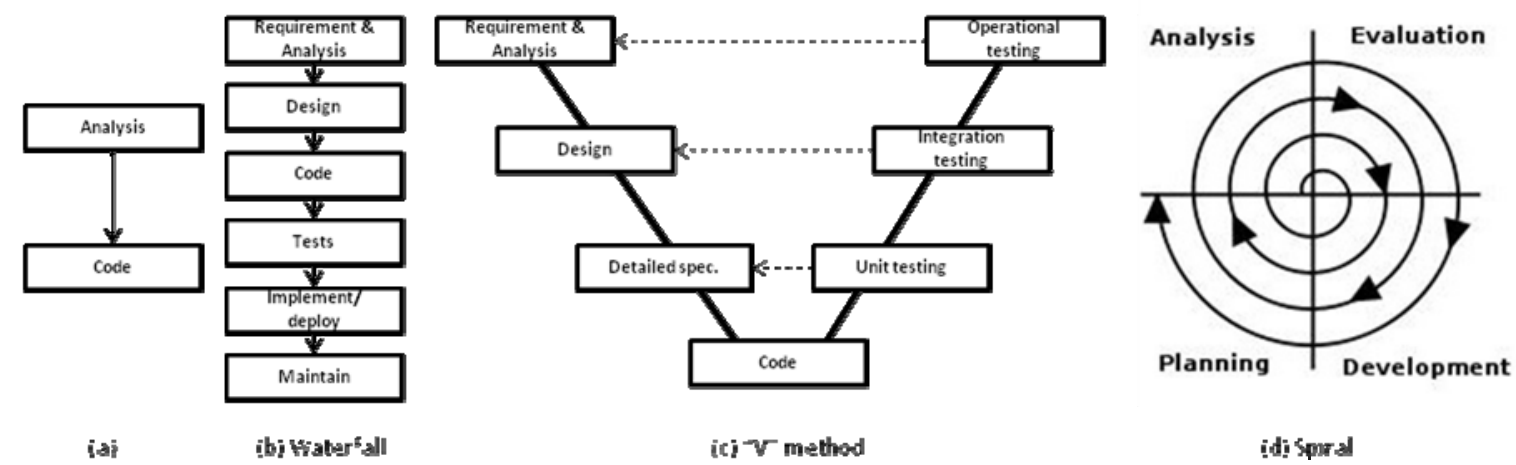

Figure 2. From the linear modelling methodology (a) to an incremental approach (d)

The demand for tools for participatory modelling engaging a mix of customers including managers, stakeholders, scientists and other experts appeared only recently in commercial software development. The industry has provided news solutions that serve the efficiency of model building but with the sole purpose of limiting budget overruns and obtaining rapid release from customers. With this perspective, the coconstruction is viewed as serving a "modelling-driven" approach. We see that even the best approaches in software development are only a refinement of the old Waterfall method. These conventional approaches focus on the product, following pre-established requirements and again, serve a Modelling-driven approach.

In this context we must take care of drawbacks of iterative processes to address complex environmental problems. We describe thereafter a preliminary framework in case of a sustainability driven approach.

\subsection{Distinguishing Modelling Agenda from Participants Interactions}

Incremental processes are often suggested to conduct participatory modelling for socio-environmental issues. According to d'Aquino (2002), the complexity of the decision making process legitimizes an iterative approach. However, the use of an iterative process does not insure its convergence at the risk of stakeholders' fatigue. First, as in any iterative process, we can separate a continuous component from an oscillating component. The continuous part corresponds to the "story" of the participatory process, namely its Agenda. The oscillating component corresponds to the exchanges between participants. 


\section{An agenda for model building under a sustainability-driven approach}

It is important that all participants have a clear perception of the agenda that they will be driven along as the work progresses. A quantitative agenda with "deliverable steps", corresponding to the Waterfall method, is not suitable in working with stakeholders whose perception of the system evolves along the process. Concerning the uncertainties of the models, Brugnach, et al. (2008) have suggested classification of models, namely : the "Learning-Communication-Exploratory-Prediction" stages. We choose a somewhat similar classification ECOF for the "Exploratory-Communicative-Operational-Forecast" stages. Those are the steps of a model growing in complexity. The highest level of quality of a "forecast-able" model is seen in its transfer to the policy-making area and judged in terms of ownership by the managers but with a partnership maintained. These stages can be considered as fundamental steps towards addressing the environmental problem and may correspond to organized meetings with the stakeholders. Such an agenda brings to the stakeholders a better perception of the model growing in quality than a classical solution-based industrial-driven agenda (table 1) and each step correspond to a different role of the participants in the coconstruction of the model.

\begin{tabular}{|c|c|}
\hline Sustainability-driven agenda ECOF & Industrial-driven agenda \\
\hline $\begin{array}{l}\text { Exploratory : we explore the problem, this can lead } \\
\text { to a conceptual model }\end{array}$ & $\begin{array}{l}\text { Design/requirement }: \text { we analyse the problem in } \\
\text { terms of requirements for a solution }\end{array}$ \\
\hline $\begin{array}{l}\text { Communicative : a model can show numerical } \\
\text { results as a first representation of reality }\end{array}$ & $\begin{array}{l}\text { Coding/formulating: internal technical operation } \\
\text { with only a few stakeholders }\end{array}$ \\
\hline $\begin{array}{l}\text { Operational : the model shows the most suitable } \\
\text { representation (calibrated) of what seems to be the } \\
\text { problem to address; allows for scenarios }\end{array}$ & $\begin{array}{l}\text { Testing: internal \& external testing as an appraisal } \\
\text { step in order to obtain release from actors / } \\
\text { stakeholders }\end{array}$ \\
\hline $\begin{array}{l}\text { Forecast : the model can be operated by managers } \\
\text { in policy-making context for scenario testing; it is } \\
\text { the most advanced stage, degree of ownership by } \\
\text { users measuring the quality of the product; } \\
\text { partnership with developers is maintained... }\end{array}$ & $\begin{array}{l}\text { Deploy: the model and/or its' results are transmitted } \\
\text { for use; possibility of a new design. }\end{array}$ \\
\hline
\end{tabular}

Table 1 : The ECOF agenda for model development vs. Industrial driven agenda

In some study sites, the SPICOSA project has tested this approach (Balle-Beganton et al. 2010). The use of the system-oriented platform ExtendSim has allowed for a quality growing process from conceptual models with empty-boxes of the systems to communication-able and operative models (figure 3 ).

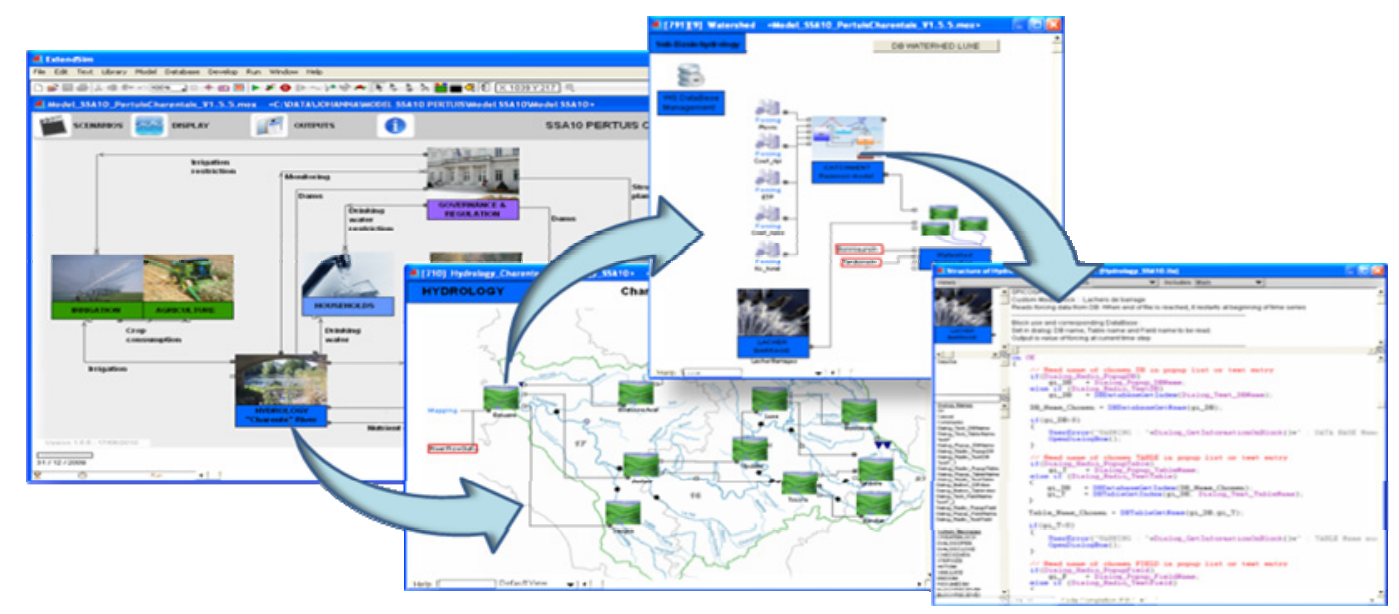

Figure 3. Iterative building of a system model under ExtendSim platform, Pertuis Charentais Study Site, SPICOSA Integrated Project

ECOF agenda allow us to oppose two distinct dimensions of the building process (figure 4): the "functionality" dimension expresses the increase of the model functionalities. The "quality" dimension expresses the ability of the model to correctly achieve these functionalities, i.e. to correctly take account all the different inputs of its functions and procedures. 
The classical industrial approach (Waterfall) leads to a fast growing quality of the process at its beginning (requirement analysis, coding libraries). However the functionalities are only expressed at the end of the process by linking all the elements together. Meeting the sharp target depends only on the quality at the beginning of the process. In this sense, this is as a "ballistic" approach because the controllability of the process only exists at its beginning (high slope of the curve). If the target is missed, the process must be restarted almost at its beginning.

With ECOF, we encourage a low-growing quality at the beginning of the process, implementing much functionality but with poor quality. Socioecological issues may differ from industrial problems in that their target is often a "fuzzy target". As long as the issue is not completely explored, the process can't express a "sharp

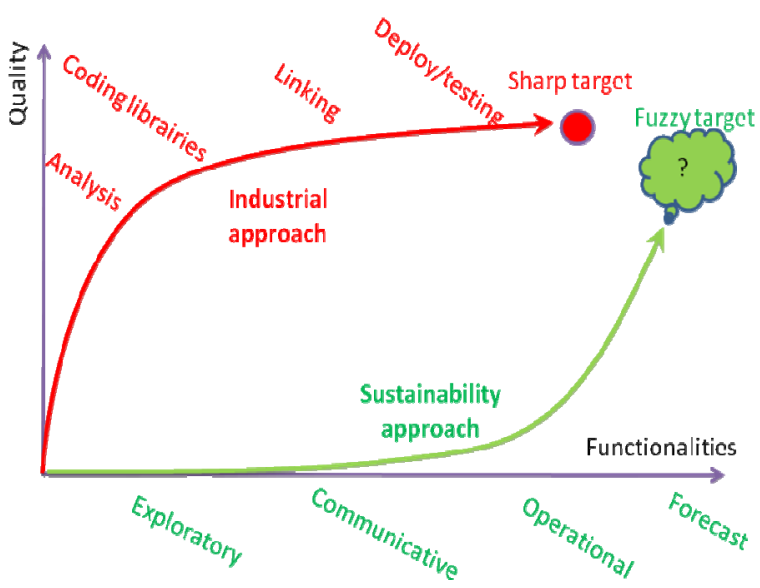

Figure 4. Industrial vs. sustainability-driven approaches for model building. The controllability of the process corresponds to the slope of each curve and appears at the end of a sustainability-driven approach. target". The increased growth of the "Functionalities/Quality" curve appears only in the end. This allows for a good controllability of the process as the target progressively gets sharper.

\section{Interaction pendulum}

The Agenda described above gives the continuous part of the incremental process. The oscillating part corresponds to the exchanges among participants around the model building. Theses exchanges must remain maintained and cyclic. They must be structured to avoid the drift of the process along the ECOF agenda and ensure the global quality of the co-construction process. A quality control namely "Plan-DoCheck-Act" (Deming 1950) used in ISO norm for industrial processes could be used but is designed solely for internal use and must be adapted to model co-construction with stakeholders.

We saw that the major problem of co-constructing models lies in the perception of the model by the actors, and the perception of their expertise. Modelling (numerical or not) always goes from a level of "concept" to a level of "reality". Conceptualisation and Realisation are two exclusive and dual operations. For example: a "design" step is a "conceptualisation" operation of a certain reality perceived by modellers. A Design is followed by a Formulation step corresponding to the "realization" of this concept. This reality is presented to stakeholders (or other actors) during an Output step which corresponds to a "conceptualization" of the presented outputs by stakeholders. Finally, the stakeholder feedback is expressed by an Appraisal step in which the stakeholders expose their perception of the model (realization), giving a new design and initiating a new cycle.

In physical sciences, examples of such transfers of dual quantities are well known: tension and current in electricity, pressure and speed in acoustic waves etc. The exchanges between dual quantities can lead to periodic and maintained oscillations with minimum energy. These oscillations will sustain the dynamics between the modellers and stakeholders. This DFOA protocol we present here (Design-FormulationOutput-Appraisal) is well balanced because it always corresponds to periodic exchanges (like a pendulum), between two dual quantities: the concept and its realization (figure 5). SPICOSA suggests such a four-stage control loop (Design-Formulation-Appraisal-Outputs) (Tett et al., 2011) but we argue that in SPICOSA the inversion of "appraisal" followed by the "outputs" is a cause of drift of the construction because of its non well-balanced character, the possible auto-appraisal from modeller part (as we observe it) and the "waterfall-like" structure focused on the "product" (the "outputs" of the model).

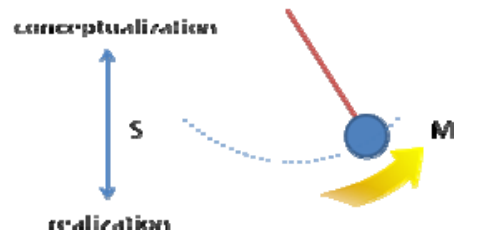

Design

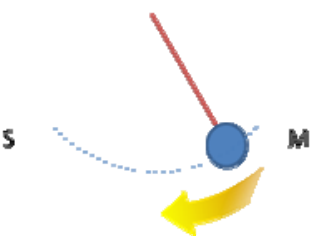

Formulation

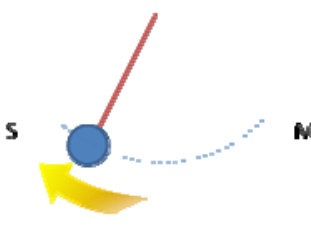

Outputs

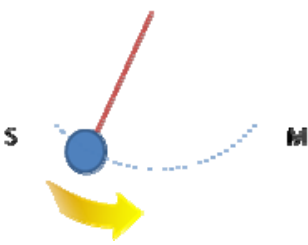

Appraisal

Figure 5. Pendulum effect between modelers (M) and stakeholders (S) along the DFOA protocol. 


\section{CONCLUSION}

The sustainability-driven approach presented here is a method based on a more qualitative than quantitative Agenda for model development as part of a larger social process, privileging the exploration of fuzzy objectives and accepting a slow growing quality of the model itself. This is intended to avoid the common biases and consequences of solution oriented developments based on initially well identified problems. . The exchanges among participants about the model are then a key concern. To maintain this engagement and ensure that the model develops to the most advanced stages, the social process involved needs a formal protocol. We suggest that it could be in four steps under an iterative protocol Design Formulation Output Appraisal inspired from control quality procedures. This is intended to sustain a pendulum like momentum between modellers and stakeholders and to constitute a quality context to avoid the drift of the process. Experience shows that a sustainability-driven approach requires an Architect. The Architect role is different from a project manager or a facilitator who may also be needed. It has to be a driving force in maintaining over time the dynamic of the process. This Architect is not a predefined function for a scientist, a manager, a policy-maker or even a modeller. Anybody as long as this leadership function is recognized by the group can play the role. But it's clear identification is crucial.

\section{REFERENCES}

Argyris, C. (1999) On organizational learning. Wiley-Blackwell. Oxford. UK. 464 pp

Aquino Patrick, Christophe Le Page, Francois Bousquet and Alassane Bah, A novel mediating participatory modelling: the self-design process to accompany collective decision making, International Journal of Agricultural Resources, Governance and Ecology, 2002, vol. 2, issue 1, pages 59-74

Ballé-Béganton J., Lample M., Bacher C., Fiandrino A., Guillard V., Laugier T., Mongruel R., Pérez Agúndez J.A. (2010) A modelling platform for complex socio-ecosystems: an application to freshwater management in coastal zones, IEMSS Fifth Biennial Meeting, 5-8 July 2010, Ottawa, Canada.

Boehm B, "A Spiral Model of Software Development and Enhancement", ACM SIGSOFT Software Engineering Notes", "ACM", 11(4):14-24, August 1986

Bosworth Mike, (1993) Solution Selling: Creating Buyers in Difficult Selling Markets, McGraw-Hill

Brugnach, M., Pahl-Wostl, C., Lindenschmidt, K. E., Janssen, J. A. E. B, Filatova, T., Mouton, A., Holtz, G., van der Keur, P., Gaber, N., Complexity and Uncertainty: Rethinking the Modelling Activity, in Environmental modelling, software and decision support, Jakeman, A. J. et al. (eds.), vol. 3, Amsterdam, 49-66, 2008.

Burgelman, R. A. (Mar.,1994) Fading Memories: A Process Theory of Strategic Business Exit in Dynamic Environments. Administrative Science Quarterly Vol. 39, No. 1, pp. 24-56

Checkland, P. B. (1981) Systems Thinking, Systems Practice. Wiley. Chichester.

Deming, W.E. (1950) Elementary Principles of the Statistical Control of Quality, JUSE

Hage M ; Leroy P - Petersen AC (eds) (13 march 2008), Stakeholder Participation Guidance for the Netherlands Environmental Assessment Agency: Main Document, PBL Netherlands Environmental Assessment Agency, Report No. 550032007

Benington, Herbert D. (1 October 1983). "Production of Large Computer Programs". IEEE Annals of the History of Computing (IEEE Educational Activities Department) 5 (4): 350-361.

Gregory, Frank Hutson, 1951- (1993) A logical analysis of soft systems modelling: implications for information system design and knowledge based system design. PhD thesis, University of Warwick.

Janssen, Judith A.E.B. and Hoekstra, Arjen Y. and Kok de, Jean-Luc and Schielen, Ralph M.J. (2009) Delineating the Model-Stakeholder Gap: Framing Perceptions to Analyse the Information Requirement in River Management. Water Resources Management, 23 (7). pp. 1423-1445. ISSN 0920-4741

L'Etang Jacquie, Magda Pieczka (2006) Public relations: critical debates and contemporary practice Stirling Media Research Institute, University of Stirling, Scotland. Lawrence Erlbaum Associates

McIntosh, B.S., Giupponi, C. and Voinov, A.A. (2008) Bridging the gaps between design and use : developing tools to support environmental management and policy. In: Environmental modelling, software and decision support: state of the art and new perspectives ed. by A.J. Jakeman, A.A. Voinov, [et al.]. - Amsterdam: Elsevier, 2008. (Developments in integrated environmental assessment; 3 ).

Tett P., A, Sanderg and A. Mette (Eds), SUSTAINING COASTAL ZONE SYSTEMS, Dunedin Academic Press. 2011, ISBN: 978-1-906716-27-1

Voinov, A.A. and Bousquet, F. (2010) Modelling with stakeholders : position paper. In: Environmental modelling and software, 25 (2010)11 pp. 1268-1281. 